\title{
Contribuições da simulação realística no ensino-aprendizagem da enfermagem:
}

\section{revisão integrativa}

\author{
Contributions of realistic simulation in nursing teaching-learning: an integrative review \\ Contribuciones de la simulación realista en la enseñanza-aprendizaje de enfermería: una revisión \\ integradora
}

Recebido: 10/02/2021 | Revisado: 17/02/2021 | Aceito: 20/02/2021 | Publicado: 28/02/2021

Isabella Domingues

ORCID: https://orcid.org/0000-0003-0764-7029

Universidade Estadual de Londrina, Brasil

E-mail: isabella.domingues@uel.br

Eleine Martins

ORCID: https://orcid.org/0000-0001-6649-9340 Universidade Estadual de Londrina, Brasil E-mail: eleinemartins@uel.br

Caroline Lourenço de Almeida

ORCID: https://orcid.org/0000-0002-6043-9301 Universidade Estadual de Londrina, Brasil E-mail: caroline_lat@hotmail.com

Daniel Augusto da Silva

ORCID: https://orcid.org/0000-0002-2716-6700

Fundação Educacional do Município de Assis, Brasil E-mail: daniel.augusto@unifesp.br

\begin{abstract}
Resumo
O objetivo deste estudo foi analisar a contribuição da simulação realística do ensino de graduação em Enfermagem. Trata-se de um estudo de revisão integrativa, com abordagem qualitativa, com dados coletados por meio da busca de artigos anexados nas bases de dados referentes à Biblioteca Virtual em Saúde e Literatura Latino-Americana e do Caribe em Ciências da Saúde. A combinação dos descritores na pesquisa foi de simulação ou treinamento por simulação e enfermagem, e período de busca foi realizado de janeiro a março de 2020. O total de material bibliográfico dessa revisão foi de 34 artigos. A maioria dos artigos são de natureza metodológica, e método de pesquisa com instrumento avaliativo de pré e pós teste com prática. As principais vantagens citadas pelos periódicos em relação à utilização da simulação realística foram: facilitar o conhecimento; autoconfiança e precisão; aproximação da realidade e raciocínio clínico. Esse estudo demonstrou que a simulação realística em enfermagem, vem se tornando um instrumento-chave e inovador de aprendizado. É uma ferramenta capaz de favorecer a retenção do conhecimento com o desenvolvimento de raciocínio clínico, segurança e precisão nas habilidades.
\end{abstract}

Palavras-chave: Simulação; Educação em enfermagem; Ensino; Aprendizagem.

\begin{abstract}
The aim of this study was to analyze the contribution of realistic simulation of undergraduate nursing education. This is an integrative review study, with a qualitative approach, with data collected through the search for articles attached in the databases referring to the Virtual Health Library and Latin American and Caribbean Literature in Health Sciences. The combination of descriptors in the research were simulation or simulation and nursing training, and the search period was carried out from January to March 2020. The total bibliographic material of this review was 34 articles. Most of the articles are of a methodological nature, and a research method with a pre- and post-test evaluation tool with practice. The main advantages cited by the journals in relation to the use of realistic simulation were: to facilitate knowledge; self-confidence and precision; approximation of reality and clinical reasoning. This study demonstrated that realistic nursing simulation is becoming a key and innovative learning tool. It is a tool capable of promoting the absorption of knowledge with the development of clinical reasoning, safety and precision in skills.
\end{abstract}

Keywords: Simulation technique; Education nursing; Teaching; Learning.

\section{Resumen}

El objetivo de este estudio fue analizar la contribución de la simulación realista de la formación de pregrado en enfermería. Se trata de un estudio de revisión integradora, con enfoque cualitativo, con datos recolectados a través de la búsqueda de artículos adjuntos en las bases de datos referentes a la Biblioteca Virtual en Salud y Literatura Latinoamericana y Caribeña en Ciencias de la Salud. La combinación de descriptores en la investigación fueron 
simulación o simulación y formación en enfermería, y el período de búsqueda se realizó de enero a marzo de 2020 . El material bibliográfico total de esta revisión fue de 34 artículos. La mayoría de los artículos son de naturaleza metodológica y un método de investigación con una herramienta de evaluación previa y posterior a la prueba con práctica. Las principales ventajas citadas por las revistas en relación al uso de simulación realista fueron: facilitar el conocimiento; autoconfianza y precisión; aproximación de la realidad y razonamiento clínico. Este estudio demostró que la simulación de enfermería realista se está convirtiendo en una herramienta de aprendizaje clave e innovadora. Es una herramienta capaz de promover la absorción de conocimientos con el desarrollo del razonamiento clínico, seguridad y precisión en las habilidades.

Palabras clave: Simulación; Educación en enfermería; Enseñanza; Aprendizaje.

\section{Introdução}

O ensino tradicional realizado com a estratégia de aulas expositivas vem sofrendo constantes mudanças nas últimas décadas, principalmente na área da saúde. As instituições de ensino passam então, a implementar novas estratégias complementares para sanar as lacunas no conhecimento, quando as aulas são apenas teóricas. A nova abordagem busca associar teoria à prática, por meio de uma metodologia que reúne a utilização de cenários controlados, tornando o ambiente mais próximo do real, conhecida como simulação realística (Yamane, Machado, Osternack, \& Mello, 2019).

No treinamento utilizando a simulação realística é estabelecido um ambiente situacional, criado para realizar técnicas com o objetivo de aprender, melhorar competências, ensaiar e realizar processos avaliativos de determinado ambiente real, seja com ações humanas ou por meio de sistemas (Sebold, Böell, Girondi, \& Santos, 2017).

A simulação realística permite que o estudante aprenda o conteúdo de forma aproximada do ambiente real e com perfil ético e seguro. É uma estratégia de alta fidelidade de capacitação, produção, desenvolvimento do conhecimento e habilidades psicomotoras. Ela proporciona às equipes multiprofissionais a realização de diferentes abordagens dos cenários na prática clínica, e, é possível utilizá-la diversas vezes para que os objetivos de aprendizagem sejam alcançados (Miranda, Mazzo, \& Pereira Junior, 2018).

O preparo do método da simulação realística é caracterizado pela elaboração de conteúdo e planejamento estratégico para abordagem temática, que pode ser aplicada por meio de diversos recursos caracterizando a baixa, média ou alta complexidade (Ferreira, Carvalho, \& Carvalho, 2015).

Os manequins que permitem programação prévia, como fala e sinais vitais, são dispositivos classificados em alta fidelidade. Já os de média fidelidade oferecem sons respiratórios, cardíacos e pulsação. Os de baixa fidelidade estão classificados em manequins estáticos, para habilidades específicas como sondagem, ou peças anatômicas que proporcionam técnicas com segurança como punções (Ferreira, Carvalho, \& Carvalho, 2015).

Segundo os mesmos autores, uma pessoa previamente treinada também pode ser protagonista e intérprete do paciente, isso facilita a interação entre paciente-equipe. Há também a simulação híbrida, composta pelo ator juntamente de uma peça anatômica para procedimentos invasivos.

O uso da simulação como ferramenta de ensino garante diversos benefícios, tais como a repetição de técnicas e habilidades quantas vezes forem necessárias, oportunidade de correção de falhas, proporciona experiências do ambiente real, discussões sobre dificuldades encontradas e oportunidade de um ensino ético e profissional, além de segurança ao paciente, o qual é essencial, uma vez que o acometimento de falhas na realidade pode resultar em incapacidades, lesões ou até mesmo ser fatal (Melo et al., 2016; Fabri et al., 2017).

Dessa forma, para elaboração desse trabalho, buscou-se responder a seguinte questão norteadora como foco principal deste trabalho: $\mathrm{O}$ que encontramos na literatura referente ao processo aprendizagem em enfermagem utilizando a simulação realística?

Considerando a importância da simulação realística no ensino aprendizagem, o objetivo deste estudo foi analisar a contribuição da simulação realística do ensino de graduação em Enfermagem. 


\section{Metodologia}

Trata-se de um estudo de revisão integrativa, com abordagem qualitativa. A revisão integrativa configura-se, portanto, como um tipo de revisão da literatura que reúne achados de estudos desenvolvidos mediante diferentes metodologias, permitindo aos revisores sintetizar resultados sem ferir a filiação epistemológica dos estudos empíricos incluídos (Soares et al., 2014).

Os dados foram coletados por meio da busca de artigos anexados nas bases de dados referentes à Biblioteca Virtual em Saúde (BVS) e Literatura Latino-Americana e do Caribe em Ciências da Saúde (LILACS). A combinação dos descritores na pesquisa foi de simulação ou treinamento por simulação e enfermagem, de acordo com os Descritores em Ciências da Saúde (DeCs). A escolha dos descritores foi de acordo com o objetivo da pesquisa, que é analisar a contribuição da simulação realística no ensino de graduação da enfermagem. O período de busca foi realizado de janeiro a março de 2020, pelos autores da pesquisa.

Foram aplicados os seguintes filtros para critério de inclusão: treinamento por simulação como assunto principal, idioma no português e intervalo de publicação nos últimos cinco anos. Os critérios de exclusão foram: texto não disponível na íntegra e a não abordagem da simulação realística como estratégia de ensino para a enfermagem.

A pesquisa na BVS com os descritores de simulação ou treinamento por simulação e enfermagem, resultou em 805 artigos, porém, quando aplicados os critérios de inclusão e exclusão totalizou-se em 24 artigos. No LICACS a busca com os descritores simulação realística ou treinamento por simulação e enfermagem, resultou em 19 artigos. Com os critérios de inclusão e exclusão, restaram 14 artigos. O total de material bibliográfico dessa pesquisa foi de 34 artigos, quatro artigos estavam presentes nas duas plataformas de dados. A Figura 1 demonstra o processo de seleção dos artigos.

Figura 1. Processo de seleção dos artigos que compõem esta revisão integrativa. Londrina, PR, 2020.

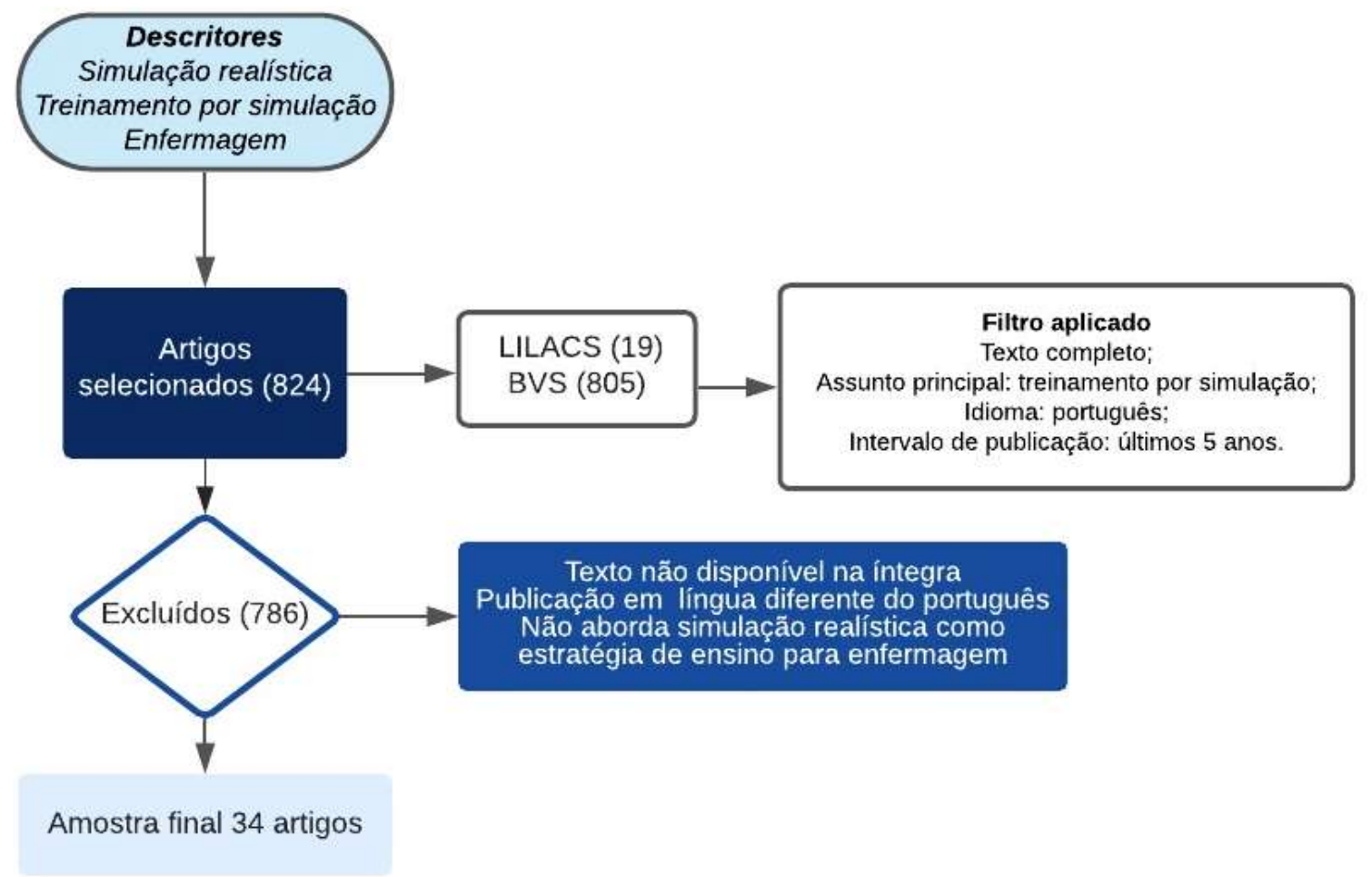

Fonte: Autores.

Foi elaborado uma tabela no Excel 2007 para tabulação e organização dos dados: título, periódico, ano de publicação, estado, autores, objetivo da pesquisa, natureza do estudo, método de análise, método de pesquisa, etapas do estudo, principais resultados, conclusão e dicas para os próximos estudos. Os artigos passaram por uma leitura minuciosa durante quatro meses 
pelos autores e foram adicionados na tabela de dados para análise.

\section{Resultados}

A publicação dos artigos selecionados ocorreu com maior frequência (35,29\%) no ano de 2017. Em 2018 houve publicação de 29,41\% dos artigos, enquanto no ano de 2019 houve uma queda de publicação, chegando a 17,65\% dos artigos.

Segue na Tabela 1 a distribuição do número de artigos referentes a simulação publicados por revista.

Tabela 1 - Número de artigos publicados por revista, com a temática de ensino de simulação realística em enfermagem, nos últimos cinco anos, Londrina - PR, 2020.

\begin{tabular}{lrr}
\hline Periódicos & n & \multicolumn{1}{c}{$\%$} \\
\hline Revista Brasileira de Enfermagem - REBEn & 7 & 20,59 \\
Revista de Enfermagem UFPE on line & 6 & 17,65 \\
Revista Latino-Americana Enfermagem & 3 & 8,82 \\
Revista Mineira de Enfermagem & 2 & 5,88 \\
Revista da Escola de Enfermagem da USP & 2 & 5,88 \\
Revista da Rede de Enfermagem do Nordeste & 2 & 5,88 \\
Revista de Enfermagem do Centro-Oeste Mineiro & 2 & 5,88 \\
Revista Gaúcha de Enfermagem & 1 & 2,94 \\
Educação em ciência da saúde & 1 & 2,94 \\
Online brazilian journal of nursing [internet] & 1 & 2,94 \\
Revista Eletrônica de Comunicação, Informação e Inovação e Saúde. & 1 & 2,94 \\
Revista Cuidarte & 1 & 2,94 \\
Revista SOBECC & 1 & 2,94 \\
Revista do Colégio Brasileiro de Cirurgiões & 1 & 2,94 \\
Acta Bioethica & 1 & 2,94 \\
Clinical e Biomedical Research & 1 & 2,94 \\
Dissertação de Mestrado & 1 & 2,94 \\
Total & $\mathbf{3 4}$ & $\mathbf{1 0 0 , 0 0}$ \\
\hline
\end{tabular}

Fonte: Autores.

A amostra do estudo foi composta de 34 artigos com predomínio da Revista Brasileira de Enfermagem (20,59\%), da Revista de Enfermagem UFPR online (17,65\%) seguida da Revista Latino-americana de Enfermagem (8,82\%).

Segue na tabela dois a distribuição da natureza do estudo das publicações encontradas para análise.

De acordo com a Tabela 2, a maior parte dos estudos adotou-se como natureza de estudo o metodológico (14,71\%), seguido da revisão, relato de experiência, quase experimental do tipo pré e pós teste e quase experimental, todos com $11,76 \%$ dos artigos selecionados.

Considerando o método de análise, a revisão apresentou que 47,07\% dos estudos foram quantitativos, 41,17\% foram qualitativos e 11,76\% foram classificados em quanti-quali. Segue na tabela três a distribuição da análise dos resultados. 
Tabela 2 - Número de periódicos com abordagem de simulação realística em enfermagem, relacionado a natureza do estudo, Londrina - PR, 2020.

\begin{tabular}{lrr}
\hline Natureza do estudo & n & \multicolumn{1}{c}{$\%$} \\
\hline Metodológico & 5 & 14,71 \\
Quase experimental, pré e pós teste & 4 & 11,76 \\
Relato de experiência & 4 & 11,76 \\
Revisão & 4 & 11,76 \\
Transversal & 3 & 8,82 \\
Quase experimental & 3 & 8,82 \\
Exploratório e descritivo & 2 & 5,88 \\
Pesquisa - ação & 2 & 5,88 \\
Ensaio reflexivo & 2 & 5,88 \\
Ensaio clínico randomizado & 1 & 2,94 \\
Experimental, temporal prospectivo & 1 & 2,94 \\
Coorte, retrospectivo & 1 & 2,94 \\
Retrospectivo, quase experimental & 1 & 2,94 \\
Documental, retrospectivo & 1 & 2,94 \\
Total & $\mathbf{3 4}$ & $\mathbf{1 0 0 , 0 0}$ \\
\hline
\end{tabular}

Fonte: Autores.

Tabela 3 - Número de periódicos com abordagem de simulação realística em enfermagem, relacionado com método de pesquisa utilizado para aplicar o estudo, Londrina - PR, 2020.

\begin{tabular}{lrr}
\hline Método de pesquisa & n & \multicolumn{1}{c}{$\%$} \\
\hline Instrumento avaliativo de pré e pós teste com prática & 16 & 47,06 \\
Revisão bibliográfica & 5 & 14,71 \\
Comparação de grupos & 4 & 11,76 \\
Elaboração de roteiro & 3 & 8,82 \\
Adaptação de instrumento & 2 & 5,88 \\
Observação & 2 & 5,88 \\
Análise retrospectiva & 1 & 2,94 \\
Elaboração de instrumento & 1 & 2,94 \\
Total & 34 & 100,00 \\
\hline
\end{tabular}

Fonte: Autores.

Dos periódicos selecionados 47,06\% utilizaram instrumento avaliativo pré e pós simulação realística como método de pesquisa.

Dos temas abordados para a simulação realística a urgência e emergência foi o cenário mais explorado $(17,65 \%)$ para desenvolvimento de estudos. As situações de segurança do paciente e habilidades de enfermagem foram adotadas para a implementação da simulação em 14,71\% das situações cada uma. Entretanto, os cenários de consulta de enfermagem e contribuição para simulação, apresentaram-se igualmente distribuídos em 11,76\% dos estudos. A saúde mental, instrumento de simulação e tecnologia foram citados em 5,88\% das pesquisas para treinamento de habilidades, respectivamente. A abordagem de simulação com implementação na área de saúde do adulto, resíduos, Peri operatória e obstetrícia, foram igualmente distribuídos em $2,94 \%$ dos artigos.

Segue na Tabela quatro as vantagens referidas pelos autores dos periódicos selecionados. 
Tabela 4 - Principais vantagens citadas pelos periódicos em relação à utilização da simulação realística, Londrina - PR, 2020.

\begin{tabular}{lrr}
\hline Principais vantagens da simulação realística & n & \multicolumn{1}{c}{$\%$} \\
\hline Facilitador do conhecimento & 8 & 12,50 \\
Autoconfiança e precisão & 6 & 9,38 \\
Aproximaça da realidade & 5 & 7,81 \\
Raciocínio clínico & 5 & 7,81 \\
Desenvolvimento de competências e habilidades & 4 & 6,25 \\
Fortalecer teoria à prática & 4 & 6,25 \\
Segurança do Paciente & 3 & 4,69 \\
Rever falhas & 3 & 4,69 \\
Tomada de decisões & 3 & 4,69 \\
Atender o objetivo de aprendizagem & 2 & 3,13 \\
Fixação de conteúdo & 2 & 3,13 \\
Ferramenta complementar no processo de ensino & 2 & 3,13 \\
Refletir sobre futura situação & 2 & 3,13 \\
Estratégia de desenvolvimento profissional & 2 & 3,13 \\
Ferramenta de comunicação e de apoio ao docente & 1 & 1,56 \\
Inovador & 1 & 1,56 \\
Processo complexo, necessita de abertura a sugestões e adaptações & 1 & 1,56 \\
Aumento de fatores estressores relacionados à falta de competências; & 1 & 1,56 \\
Autoavaliação & 1 & 1,56 \\
Integração de cenários & 1 & 1,56 \\
Excelência no ensino e atendimento & 1 & 1,56 \\
Estratégia pedagógica & 1 & 1,56 \\
Apoio do professor durante a prática & 1 & 1,56 \\
Conscientização na performance no atendimento & 1 & 1,56 \\
Educação permanente em enfermagem & 1 & 1,56 \\
Estratégia eficaz de ensino e aprendizagem & 1 & 1,56 \\
Prática formativa nos processos de formação da enfermagem & 1 & 1,56 \\
Total & $\mathbf{6 4}$ & $\mathbf{1 0 0 , 0 0}$ \\
\hline & & \\
\hline & 13 &
\end{tabular}

Fonte: Autores.

No segmento referente às vantagens e desvantagens relacionadas ao emprego da simulação realística, foram citados como benefícios ser um facilitador do conhecimento (12,50\%), autoconfiança e precisão (9,38\%), assim como o raciocínio clínico $(7,81 \%)$ e fortalecedor da teoria à prática $(6,25 \%)$. A simulação foi referida, sobretudo, como ferramenta para o desenvolvimento de competências e habilidades (6,25\%). Nesta vertente, foram citados: tomada de decisões $(4,69 \%)$; chance de rever falhas (4,69\%), segurança do paciente (4,69\%), estratégia de desenvolvimento profissional $(3,13 \%)$ e reflexão sobre futura situação $(3,13 \%)$.

Já no campo das dificuldades encontradas durante a simulação realística 42,86\% apresentaram a amostra insuficiente como uma desvantagem. A necessidade de mais estudos sobre a temática foi citada em 19,05\% dos artigos. Fatores estressores e falta de tecnologia na simulação apareceram com 9,52\% e 4,76\% respectivamente.

Segue na Tabela 5 as principais recomendações dos artigos estudados. 
Tabela 5 - Principais recomendações dos estudos sobre simulação realística na enfermagem, para futuras pesquisas, Londrina PR, 2020.

\begin{tabular}{|c|c|c|}
\hline Recomendações para os próximos estudos & $\mathrm{n}$ & $\%$ \\
\hline Estudos e reflexões mais aprofundados acerca do tema & 5 & 26,32 \\
\hline Elaborar estratégias que permitam a minimização dos fatores estressores & 2 & 10,53 \\
\hline Pesquisa em outras escolas e lugares diferentes & 2 & 10,53 \\
\hline Adaptação nos diversos contextos & 1 & 5,26 \\
\hline Simulação de baixa fidelidade pode ser considerada baixo custo & 1 & 5,26 \\
\hline Realização de atividade prática previamente ao início do curso, além da efetuada ao final. & 1 & 5,26 \\
\hline Continuidade na construção de cenários e desenvolvimento de simulações & 1 & 5,26 \\
\hline Testar a eficácia no aumento de conhecimento teórico e prático & 1 & 5,26 \\
\hline Tipo de simulação previamente definida & 1 & 5,26 \\
\hline Treinamentos com simulação da equipe interdisciplinar e multiprofissional & 1 & 5,26 \\
\hline Pesquisas voltadas a aproximação com a realidade do trabalho & 1 & 5,26 \\
\hline $\begin{array}{l}\text { Avaliar a satisfação do aluno com a estratégia e reforçar a importância de a simulação ser inserida na grade } \\
\text { curricular }\end{array}$ & 1 & 5,26 \\
\hline Construção de um roteiro teórico-prático de cenário simulado & 1 & 5,26 \\
\hline Total & 19 & 100,00 \\
\hline
\end{tabular}

Fonte: Autores.

Das principais recomendações para futuras pesquisas apenas $55,88 \%$ das pesquisas propuseram sugestões, as mais citadas foram o desenvolvimento de estudos e reflexões mais aprofundados no tema, devido a carência de periódicos em simulação realística na enfermagem $(26,32 \%)$. Também foi proposto elaborar mais estratégias que possam diminuir os fatores estressores nos estudantes $(10,53 \%)$ e realizar futuras pesquisas em lugares diferenciados $(10,53 \%)$.

\section{Discussão}

A simulação realística tem como proposta ser ferramenta inovadora e complementar no ensino da enfermagem. É uma tecnologia diferenciada que qualifica o ensino, seja ele referente a uma situação clínica, como também situações de risco à saúde. Isso garante que o estudante desenvolva habilidades e possibilita o docente a detectar previamente prováveis situações de enfrentamento. No presente estudo diversas vantagens foram encontradas, como o aumento da retenção do conteúdo, desenvolvimento de segurança nas práticas e aplicação do raciocínio clínico em um ambiente fictício (Salvador, Martins, Alves, Pereira, Santos, \& Tourinho, 2015).

Com o intuito de oferecer aos estudantes treinamentos seguros e controlados com embasamento teórico, a análise com maior prevalência nas pesquisas foi a metodológica para desenvolver suas simulações. Dal Sasso et al., (2015) desenvolveram um roteiro de simulação realística em enfermagem na Universidade Federal de Santa Catarina, que se utilizou como a natureza de estudo o metodológico, o qual ocorreu por meio de buscas teóricas sobre conceito de aprendizagem.

A utilização de pré e pós teste ocorreu na maioria dos estudos analisados (47,06\%). Com o instrumento, os estudantes passaram por uma análise prévia à simulação e outra posterior, para a identificação dos acertos, dúvidas e dificuldades. Um estudo realizado em uma universidade pública na região Sul do Brasil, sobre operacionalização da simulação, demonstrou que esse tipo de instrumento de avaliação é estabelecido por orientações básicas previamente à simulação, com o intuito de instruir a atuação no cenário simulado. O desfecho da prática é determinado a partir da intervenção do estudante na cena. Após a simulação os pós-teste compreenderam em um momento de reflexão e discussão entre professor e aluno sobre pontos positivos e negativos. Consistiu em um momento crucial para as pesquisas, pois é nesta fase que é identificado as faltas de competências a serem atingidas (Oliveira, Massaroli, Martini, \& Rodrigues, 2018).

As análises quantitativas proporcionaram aos pesquisadores do atual estudo a identificação das falhas e obstáculos produzidos no ambiente simulado e a oportunidade de reflexões sobre as dificuldades encontradas. Esse método de análise 
quantitativa ofereceu ao pesquisador fundamentos às hipóteses pré-estabelecidas, identificação de falhas através das margens de erros e inúmeras oportunidades de replicações das dificuldades encontradas (Serapioni, 2000).

Acerca dos cenários utilizados no atual estudo, os ambientes que apresentaram maior exploração foram urgência e emergência, segurança do paciente, habilidades e consulta de enfermagem. Esses cenários possuem extrema importância de treinamento prévio, seja para prevenção de danos como, por exemplo, em urgência e emergência, o enfermeiro precisa agir de maneira rápida, eficaz e com precisão, sendo intolerável de erros. Já nos cenários da segurança do paciente, habilidades e consulta de enfermagem, o enfermeiro precisa estar atento às causas adversas que fazem parte do cotidiano e desempenhar a promoção da segurança do paciente.

Nesses cenários é possível alcançar os objetivos já citados previamente no estudo, como o aumento da autoconfiança e precisão, tomada de decisões e desenvolvimento profissional. Em relação aos cenários explorados em um estudo de revisão integrativa (Barreto, Silva, Moreira, Silva, \& Magro, 2014), foi apresentado a unidade de terapia intensiva como o cenário mais explorado na simulação realística, seguido de pediatria, saúde mental e centro cirúrgico. Esses cenários são ambientes próprios para práticas e reflexão de situações-problemas estabelecidos pelos profissionais, são importantes para o ciclo de conhecimentos do estudante, devido a possibilidade de diálogo teórico-prático (Santos \& Leite, 2010; Silva, Almeida, Capellini, \& Silva, 2020).

Na pesquisa de Barreto et al., (2014), seu estudo demonstrou que há uma carência de investimento nos recursos materiais e capacitação de profissionais estão entre as poucas desvantagens citadas sobre o uso do método de simulação realística. Corroborando com o estudo atual que apresentou as mesmas dificuldades, juntamente com fatores estressores dos estudantes e a amostra insuficiente de referencial teórico. Por ser uma técnica recente, as instituições ainda estão em fase de adaptação do método de simulação, as pesquisas vêm demonstrando maior número de vantagens na aplicação, se comparado com as desvantagens.

\section{Considerações Finais}

Esse estudo de revisão integrativa demonstrou que a simulação realística em enfermagem vem se tornando um instrumento-chave e inovador de aprendizado. É uma ferramenta capaz de favorecer a retenção do conhecimento com o desenvolvimento de raciocínio clínico, segurança e precisão nas habilidades. Por ser um mecanismo de identificação de erros, é essencial que o professor planeje o processo de treinamento com embasamento teórico, para que as etapas ocorram de maneira segura e eficiente para que os objetivos de aprendizagem sejam alcançados. Portanto, se faz necessário a adesão dos professores para que ocorra desenvolvimento de mais pesquisas aprofundadas sobre a vertente, pois ainda se encontra insuficiência teórica.

\section{Referências}

Barreto, D. G., Silva, K. G. N., Moreira, S. S. C. R., Silva, T. S., \& Magro, M. C. S. (2014). Simulação realística como estratégia de ensino para o curso de graduação em enfermagem: revisão integrativa. Revista Baiana de Enfermagem, 28(1), 208-214.

Dal Sasso, G. M., Sebold, L. F., Kempfer, S. S., \& Oliveira, S. N. (2015). Guia metodológico para simulação em enfermagem - CEPETEC. https://nfr.ufsc.br/files/2015/11/GUIA-METODOL\%C3\%93GICO-PARA-SIMULA\%C3\%87\%C3\%83O-EM-ENFERMAGEM-CEPETEC.pdf

Fabri, R. P., Mazzo, A., Martins, J. C. A., Fonseca, A. S., Pedersoli, C. E., Miranda, F. B. G., \& Baptista, R. C. N. (2017). Construção de um roteiro teóricoprático para simulação clínica. Revista da Escola de Enfermagem da USP, 51, e03218.

Ferreira, C., Carvalho, J. M., \& Carvalho, F. L. Q. (2015). Impacto da metodologia de simulação realística, enquanto tecnologia aplicada a educação nos cursos de saúde. Anais do Seminário Tecnologias Aplicadas a Educação e Saúde, 1(1), 32-40.

Melo, M. C. B., Magalhaes, A. M. P. B., Silva, N. L. C., Liu, P. M. F., Cerqueira Filho, L. C., \& Santos, A. F. (2016). Ensino mediado por técnicas de simulação e treinamento de habilidades de comunicação na área da saúde. Revista Médica de Minas Gerais, 26, e-1805.

Miranda, F. B. G., Mazzo, A., \& Pereira Junior, G. A. (2018). Uso da simulação de alta fidelidade no preparo de enfermeiros para o atendimento de urgências e emergências: revisão da literatura. Sci. med. (Porto Alegre, Online), 28(1), ID28675.

Oliveira, S. N., Massaroli, A., Martini, J. G., \& Rodrigues, J. (2018). Da teoria à prática, operacionalizando a simulação clínica no ensino de Enfermagem. Revista Brasileira de Enfermagem, 71(Suppl. 4), 1791-1798. 
Research, Society and Development, v. 10, n. 2, e55710212841, 2021

(CC BY 4.0) | ISSN 2525-3409 | DOI: http://dx.doi.org/10.33448/rsd-v10i2.12841

Roman, A. R., \& Friedlander, M. R. (1998). Revisão integrativa de pesquisa aplicada à enfermagem. Cogitare Enferm., 3(2), 109-112.

Salvador, P. T. C. O., Martins, C. C. F., Alves, K. Y. A., Pereira, M. S., Santos, V. E. P., \& Tourinho, F. S. V. (2015). Tecnologia no ensino de enfermagem. Revista Baiana de Enfermagem, 29(1), 33-41.

Santos, M. C., \& Leite, M. C. L. (2010). A avaliação das aprendizagens na prática da simulação em Enfermagem como feedback de ensino. Revista Gaúcha de Enfermagem, 31(3), 552-556.

Sebold, L. F., Böell, J. E. W., Girondi, J. B. R., \& Santos, J. L. G. (2017). Simulação clínica: desenvolvimento de competência relacional e habilidade prática em fundamentos de enfermagem. Rev enferm UFPE on line, 11(Supl. 10), 4184-4190.

Serapioni, M. (2000). Métodos qualitativos e quantitativos na pesquisa social em saúde: algumas estratégias para a integração. Ciência \& Saúde Coletiva, 5(1), 187-192.

Silva, D. A., Almeida, C. L., Capellini, V. K., \& Silva, R. G. (2020). Educação em enfermagem: criação de uma liga acadêmica para o ensino de urgência e emergência. Research, Society and Development, 9(3), e159932656. https://doi.org/10.33448/rsd-v9i3.2656

Soares, C. B; Hoha, L. A. K; Peduzzi, M., Sangaleti, C., Yonekura, T., \& Silva, D. R. A. D. (2014). Revisão integrativa: conceitos e métodos utilizados na enfermagem. Rev Esc Enferm USP, 48(2):335-45.

Yamane, M. T., Machado, V. K., Osternack, K. T., \& Mello, R. G. (2019). Simulação realística como ferramenta de ensino na saúde: uma revisão integrativa. Espaço para Saúde, 20(1). 